\title{
Comparison of IRIS Image Compression using JPEG 2000, SPIHT and modified SPIHT algorithm
}

\author{
${ }^{1}$ Mr. Pushkar M.Sathe ${ }^{2}$ Ms. Pranavi Mhatre \\ 1,2Electronics \&Telecommunication, SIES GST, Neru, Navi Mumbai- 400706
}

\begin{abstract}
Biometric recognition is a common and reliable way to authenticate the identity of the person. Iris recognition is an automated method of biometric identification that uses mathematical pattern-recognition techniques on images of the irides of an individual's eyes. With the growing employment of the iris recognition systems and associated research to support this, the need for large databases of iris images is growing. If the required storage space is not adequate for these images, compression is an alternative. JPEG standard cannot fulfil the advanced requirements of today's digital imagery. Digital imaging has become an integral part of the Internet and JPEG 2000 is a powerful new tool that provides power capabilities for designers and users of networked image applications. SPIHT algorithm is a very efficient technique for wavelet image compression. This is based on DWT. SPIHT is an improved and extended version of Embedded Zero tree Wavelet (EZW) coding algorithm. In this paper parameters like compression ratio, signal to noise ratio, average bit rate are obtained for JPEG 2000, SPIHT and modified SPIHT algorithms and compared.
\end{abstract}

Keywords: Biometric recognition, Compression, Discrete Transform, Quantization, Entropy Coding

\section{Iris Recognition}

In a modern world, biometric recognition is a common and reliable way to authenticate the identity of the person. A physiological characteristic is relatively stable physical characteristic such as fingerprints, iris pattern, retina scan etc. This kind of measurement is basically unchanging and unalterable during life time.[3] Biometric identification or verification of identity is currently a very active field of research. Many applications that require some degree of confidence concerning the personal identification of the people involved such as banking, computer network access or physical access to secure facility are moving away from use of paper or plastic identity cards or alpha-numeric passwords. These systems are too easy to defeat. A higher degree of confidence can be achieved by using unique physical characteristics to identify a person.

Iris recognition is an automated method of biometric identification that uses mathematical pattern-recognition techniques on video images of the irides of an individual's eyes, whose complex random patterns are unique and can be seen from some distance.

The iris is a thin circular diaphragm, which lies between the cornea and the lens of the human eye. A front view of the iris is as shown in the figure 1. The function of iris is to control the amount of light entering through the pupil and this is done by the sphincter and the dilator muscles, which adjust the size of the pupil. The average diameter of the iris is $12 \mathrm{~mm}$ and the pupil size can vary from $10 \%$ to $80 \%$ of the iris diameter.[2] Formation of iris begins during the third month of embryonic life. [5] The unique pattern on the surface of the iris is formed during the first year of life and pigmentation of stroma takes place for first few years. The density of stromal pigmentation determines the colour of the iris. The externally visible surface of the multilayered iris contains two zones, which often differ in colour. An outer ciliary zone and the inner pupillary zone and these two zones are divided by the collarets which appears as a zigzag pattern.[2]

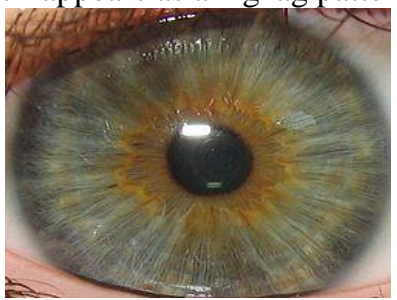

Fig. 1. A front view of human eye

Formation of the unique pattern of the iris is random and not related to any genetic factors. The only characteristic that is independent of genetics is the pigmentation of the iris which determines its colour. Due to the epigenetic nature of the iris patterns, the two eyes of an individual contain completely independent iris patterns.

A key advantage of iris recognition, besides its speed of matching and its extreme resistance to False Matches, is the stability of the iris as an internal, protected, yet externally visible organ of the eye.[2] 
Some other advantages of Iris Recognition

1) It is an internal organ that is well protected against damage and wear by a highly transparent and sensitive membrane (the cornea). This distinguishes it from fingerprints, which can be difficult to recognize after years of certain types of manual labor.

2) The iris is mostly flat, and its geometric configuration is only controlled by two complementary muscles (the sphincter pupillae and dilator pupillae) that control the diameter of the pupil. This makes the iris shape far more predictable than, for instance, that of the face.[2]

3) Even genetically identical individuals have completely independent iris textures.

4) While there are some medical and surgical procedures that can affect the colour and overall shape of the iris, the fine texture remains remarkably stable over many decades. Some iris identifications have succeeded over a period of about 30 years.

\section{Need For Compression}

In order to use biometrics for identification, the biometric data must be collected by some means. This may be a costly and time consuming process and the data obtained is valuable and must be protected. Furthermore, data collections can create an inordinate amount of data that puts a strain on the available storage. With the growing employment of the iris recognition systems and associated research to support this, the need for large databases of iris images is growing. If the required storage space is not adequate for these images, compression is an alternative. It allows a reduction in the space needed to store these iris images.

There are two types of compression schemes, lossless and lossy compression. Compression deals with techniques for reducing the space required for storage of data. The main motive of image reduction process is to remove the redundant data. In a specific area of still image compression, there are many efficient compression techniques with considerably different features.

\section{Jpeg 2000}

The JPEG standard has been in use for almost a decade now. It has proved a valuable tool during all these years, but it cannot fulfil the advanced requirements of today.[6] Today's digital imagery is extremely demanding, not only from the quality point of view, but also from the image size aspect. Current image size covers orders of magnitude,

ranging from web logos of size of less than 100

Kbits to high quality scanned images of approximate size of 40 Gbits.[1]

Digital imaging has become an integral part of the

Internet and JPEG 2000 is a powerful new tool that provides power capabilities for designers and users of networked image applications[4]

The JPEG 2000 standard provides a set of features that are of importance to many high-end and emerging applications by taking advantage of new technologies.[4] It addresses areas where current standards fail to produce the best quality or performance.

Some of the features of JPEG 2000 are

1) Superior low bit-rate performance

2) Continuous-tone and bi-level compression

3) Progressive transmission by pixel accuracy and resolution

4) Region-of-interest (ROI) coding

5) Open architecture

6) Robustness to bit errors

7) Protective image security

At the encoder, the discrete transform is first applied on the source image data. The transform coefficients are then quantized and entropy coded before forming the output code stream (bit stream). The decoder is the reverse of the encoder. The code stream is first entropy decoded, dequantized and inverse discrete transformed, thus resulting in the reconstructed image data.[4] Although this procedure looks like the one for the conventional JPEG, there are radical differences between them. 


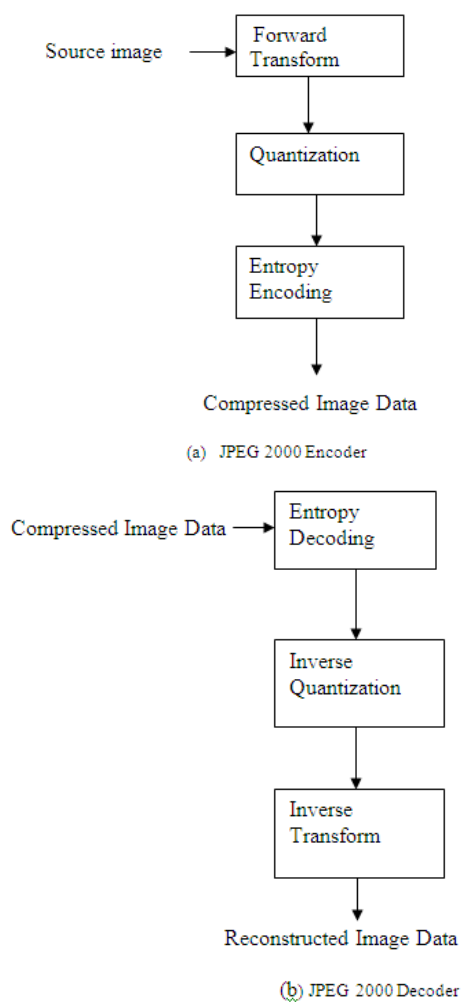

Fig 2. General block diagram of JPEG 2000

\section{Spiht}

SPIHT (Set Partitioning In Hierarchical Trees) coding algorithm is a very efficient technique for wavelet image compression. This is based on Discrete Wavelet Transform (DWT). SPIHT is an improved and extended version of Embedded Zero tree Wavelet (EZW) coding algorithm.

SPIHT is a codec which works on bitmap images. SPIHT works on the DWT of the image [8]. In a DWT of an image gets concentrated in the upper left corner, while the information content goes on reducing as we move towards the bottom right corner. As a result, pixels having maximum information content get compressed first, which leads to an accurate image reconstruction after decompression. SPIHT progressively compresses and decompresses the image which means that compression and decompression can be done to desired level. Thus SPIHT allows trade-off between size and quality [8]. The SPIHT algorithm first converts the image into its wavelet transform and then transmits information about the wavelet coefficients.

In the SPIHT algorithm, the image is first decomposed into a number of sub bands by means of hierarchical wavelet decomposition. The sub band coefficients are then grouped into sets known as spatialorientation trees, which efficiently exploit the correlation between the frequency bands. The coefficients in each spatial orientation tree are then progressively coded from the most significant bit-planes (MSB) to the least significant bit-planes (LSB), starting with the coefficients with the highest magnitude and at the lowest pyramid levels[9].

The SPIHT multistage encoding process employsthree lists and sets:

1. The list of insignificant pixels (LIP) contains individual coefficients that have magnitudes smaller than the threshold.

2. The list of insignificant sets (LIS) contains sets of wavelet coefficients that are defined by tree structures and are found to have magnitudes smaller than the threshold (insignificant). The sets exclude the coefficients corresponding to the tree and all sub tree roots and they have at least four elements.

3. The list of significant pixels (LSP) is a list of pixels found to have magnitudes larger than the threshold (significant).

4. The set of offspring (direct descendants) of a tree node, $O(i, j)$, in the tree structures is defined by pixel location $(i, j)$. The set of descendants, $D(i, j)$, of a node is defined by pixel location $(i, j)$.

$L(i, j)$ is defined as

$L(i, j)=D(i, j)-O(i, j)$.

The threshold, $T$, for the first bit-plane is equal to $2 n$, where 
$n=\log 2(\max (i, j)\{|c(i, j)|\}$

where $c(i, j)$ represents the $(i, j)$ th wavelet coefficient. All the wavelet coefficients are searched in order to obtain the maximum $c(i, j)$ after executing the discrete wavelet transform. For operations in the subsequent bit-planes of threshold $T, n$ is reduced by 1 .

For each pixel in the LIP, one bit is used to describe

its significance. If it is not significant, the pixel

remains in the LIP and no more bits are generated;

otherwise, a sign bit is produced and the pixel is

moved to the LSP. Similarly, each set in the LIS requires one bit for the significance information. The insignificant sets remain in the LIS; the significant sets are partitioned into subsets, which are processed in the same manner and at the same resolution until each significant subset has exactly one coefficient. Finally, each pixel in the LSP is refined with one bit.

The abovementioned procedure is then repeated for the subsequent resolution.

\section{Modified SPIHT}

Based on the SPIHT algorithm, two modifications are defined to develop a simpler image coding method. First modification is change in threshold value as a function of target bit rate. In order to decide whether a given pixel is significant or not, pixel value is compared with threshold [10].As per this modification, this threshold value should be subtracted by certain value depends on the target bit rate used. The value to be subtracted based on target bit rate is as given in the table no. 1.

\begin{tabular}{|c|c|}
\hline $\boldsymbol{F}$ & Rate $(\mathrm{R})$ \\
\hline 0 & $1<\mathrm{R}$ \\
\hline 2 & $0.75<\mathrm{R}<1$ \\
\hline 4 & $0.5<\mathrm{R}<0.75$ \\
\hline 6 & $0.25<\mathrm{R}<0.5$ \\
\hline 12 & $0.125<\mathrm{R}<0.25$ \\
\hline 24 & $0<\mathrm{R}<0.125$ \\
\hline
\end{tabular}

Table 1. Adaptive Quantization Parameter with corresponding target bit rate

Therefore the adaptive threshold is expressed as

$T a=(T-f)$

Where, $\mathrm{T}=$ Original value of threshold

$f=$ Adaptive Quantization Parameter

Second modification is we can discard the refinement pass, thereby decreasing memory usage and computation time with an acceptable peak signal to noise ratio (PSNR) loss, as compared to the original SPIHT[10].

\section{Methodology And Result}

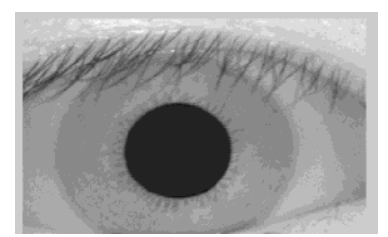

Fig 3. IRIS image from CASIA database

The images used in this research come from Chinese Academy of Sciences (CASIA) iris database.[7] This is composed of images of 108 different eyes, with 7 images of each eye (totaling 756 iris images). These images are $320 \times 2808$-bit bitmapped images (bmp), each occupying 92160 bytes on hard drive.

Software used for compression of these images using JPEG 2000 algorithm is MATLAB. Different samples are taken from the database and various parameters are obtained like average bit rate, PSNR, RMSE and Compression Ratio. Results obtained are as given in table no. 2. 


\begin{tabular}{|l|l|l|l|l|}
\hline Algorithm & $\begin{array}{l}\text { Average bit } \\
\text { rate (bpp) }\end{array}$ & $\begin{array}{l}\text { PSNR } \\
(\mathrm{dB})\end{array}$ & $\begin{array}{l}\text { Comp. } \\
\text { Ratio }\end{array}$ & RMSE \\
\hline JPEG 2000 & 0.19 & 36.1475 & 0.2702 & 3.9734 \\
\hline SPIHT & 7.4912 & 33.7947 & 0.021 & 8.3701 \\
\hline $\begin{array}{l}\text { Modified } \\
\text { SPIHT }\end{array}$ & 7.4912 & 35.3682 & 0.17 & 5.85 \\
\hline
\end{tabular}

Table 2. Results obtained Compression ratios obtained using two modifications mentioned in modified SPIHT algorithm for different values of target bit rates are as listed in table no. 3

\begin{tabular}{|c|c|}
\hline Rate & Compression Ratio \\
\hline 0.9 & 0.56 \\
\hline 0.8 & 0.5105 \\
\hline 0.6 & 0.45 \\
\hline 0.4 & 0.31 \\
\hline 0.2 & 0.25 \\
\hline 0.1 & 0.17 \\
\hline
\end{tabular}

Original IRIS image and resulted images after compression are as shown below.

Original Image
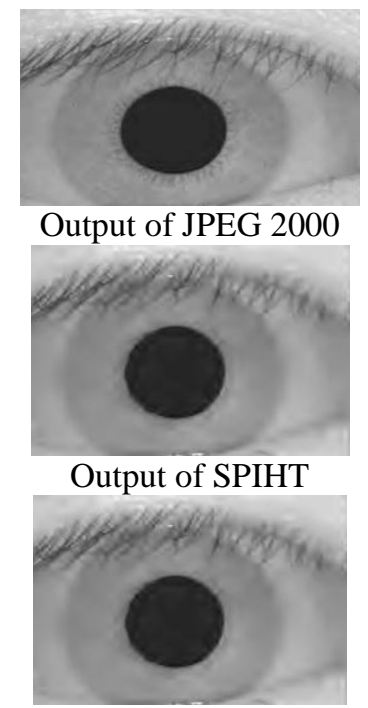

Output of modified SPIHT

Fig 4. Output images for 3 algorithms

\section{Conclusion}

This paper compares performances of three algorithms on IRIS image. SPIHT algorithm gives better compression ratio, almost 10 times than that of JPEG 2000 with an acceptable PSNR. Because of complex encoding procedure, computation time required for SPIHT is much more than JPEG 2000. But by removing refinement pass and by having variable target rates for different bit planes, it is possible to reduce computation time. This modification has allowed trade-off between computation time and compression ratio.

\section{References}

[1]. Visual Evaluation of JPEG 2000 Color Image Compression Performance,ISO/IEC JTC1/SC29/WG1 N1583, Mar. 2000.

[2]. J.Daugman, "How Iris Recognition Works" Proceedings of 2002 International Conference on Image Processing, Vol.1, 2002.

[3]. "Methods in IRIS recognition" in NCECC-09

[4]. The JPEG 2000 Still image compression standard by Athanassios Skodras, Charilaos Christopoulos, and Touradj Ebrahimi

[5]. J. Daughman. "New methods in IRIS recognition" IEEE transactions on systems. Vol.37, No.5, October 2007

[6]. M. Antonini, M. Barlaud, P. Mathieu, and I. Daubechies, "Image coding using the wavelet transform," IEEE Trans. Image Processing, pp. 205-220,

[7]. CASIA Iris Image Database. http://www.sinobiometrics.com

[8]. A simplified SPIHT algorithm by Jun-Ren Ding and Jar-Ferr Yang, Journal of the Chinese Institute of Engineers, Vol. 31, No. 4, pp. 715-719.

[9]. Said, A., and Pearlman, W. A., 1996, “A New, Fast, Set Partitioning in Hierarchical Trees," IEEE Transactions Circuits and Systems on Video Technology, Vol. 6, No. 3, pp. 243-250.

[10]. A simplified SPIHT algorithm by Jun Ren Ding and Jar Ferr Yang, Journal of the Chinese Institute of Engineers, Vol. 31, No. 4, pp. 715-719 (2008) 